\title{
Nuclear operators on Banach function spaces
}

\author{
Marian Nowak ${ }^{1}$ (1) \\ Received: 13 March 2020 / Accepted: 11 September 2020 / Published online: 6 October 2020 \\ (c) The Author(s) 2020
}

\section{Abstract}

Let $X$ be a Banach space and $E$ be a perfect Banach function space over a finite measure space $(\Omega, \Sigma, \lambda)$ such that $L^{\infty} \subset E \subset L^{1}$. Let $E^{\prime}$ denote the Köthe dual of $E$ and $\tau\left(E, E^{\prime}\right)$ stand for the natural Mackey topology on $E$. It is shown that every nuclear operator $T: E \rightarrow X$ between the locally convex space $\left(E, \tau\left(E, E^{\prime}\right)\right)$ and a Banach space $X$ is Bochner representable. In particular, we obtain that a linear operator $T: L^{\infty} \rightarrow X$ between the locally convex space $\left(L^{\infty}, \tau\left(L^{\infty}, L^{1}\right)\right)$ and a Banach space $X$ is nuclear if and only if its representing measure $m_{T}: \Sigma \rightarrow X$ has the Radon-Nikodym property and $\left|m_{T}\right|(\Omega)=\|T\|_{n u c}$ (= the nuclear norm of $T$ ). As an application, it is shown that some natural kernel operators on $L^{\infty}$ are nuclear. Moreover, it is shown that every nuclear operator $T: L^{\infty} \rightarrow X$ admits a factorization through some Orlicz space $L^{\varphi}$, that is, $T=S \circ i_{\infty}$, where $S: L^{\varphi} \rightarrow X$ is a Bochner representable and compact operator and $i_{\infty}: L^{\infty} \rightarrow L^{\varphi}$ is the inclusion map.

Keywords Banach function spaces - Mackey topologies - Mixed topologies $\cdot$ Vector measures $\cdot$ Nuclear operators $\cdot$ Bochner representable operators $\cdot$ Kernel operators . Radon-Nikodym property · Orlicz spaces · Orlicz-Bochner spaces

Mathematics Subject Classification 47B38 $\cdot$ 47B10 $\cdot 46 \mathrm{E} 30$

\section{Introduction and preliminaries}

We assume that $\left(X,\|\cdot\|_{X}\right)$ is a real Banach space. For terminology concerning Riesz spaces and function spaces, we refer the reader to $[9,13,27]$.

We assume that $(\Omega, \Sigma, \lambda)$ is a finite measure space. Let $L^{0}$ denote the corresponding space of $\lambda$-equivalence classes of all $\Sigma$-measurable real functions on $\Omega$. Then $L^{0}$ is a super Dedekind complete Riesz space, equipped with the topology $\mathcal{T}_{o}$ of con-

Marian Nowak

M.Nowak@wmie.uz.zgora.pl

1 Institute of Mathematics, University of Zielona Góra, ul. Szafrana 4A, 65-516 Zielona Góra, Poland 
vergence in measure. By $\mathcal{S}(\Sigma)$ we denote the space of all real $\Sigma$-simple functions $s=\sum_{i=1}^{n} c_{i} \mathbb{1}_{A_{i}}$, where the sets $A_{i} \in \Sigma$ are pairwise disjoint.

Let $\left(E,\|\cdot\|_{E}\right)$ be a Banach function space, where $E$ is an order ideal of $L^{0}$ such that $L^{\infty} \subset E \subset L^{1}$, and $\|\cdot\|_{E}$ is a Riesz norm on $E$. By $\mathcal{T}_{E}$ we denote the $\|\cdot\|_{E}$-norm topology on $E$. By $E^{\prime}$ we denote the Köthe dual of $E$, that is,

$$
E^{\prime}:=\left\{v \in L^{0}: \int_{\Omega}|u(\omega) v(\omega)| d \lambda<\infty \text { for all } u \in E\right\}
$$

The associated norm $\|\cdot\|_{E^{\prime}}$ on $E^{\prime}$ is defined for $v \in E^{\prime}$ by

$$
\|v\|_{E^{\prime}}=\sup \left\{\int_{\Omega}|u(\omega) v(\omega)| d \lambda: u \in E,\|u\|_{E} \leq 1\right\} .
$$

We will assume that $E$ is perfect, that is, $E=E^{\prime \prime}$ and $\|u\|_{E}=\|u\|_{E^{\prime \prime}}$. The order continuous dual $E_{n}^{\sim}$ of $E$ separates the points of $E$ and $E_{n}^{\sim}$ can be identified with $E^{\prime}$ through the Riesz isomorphism $E^{\prime} \ni v \mapsto F_{v} \in E_{n}^{\sim}$, where

$$
F_{v}(u)=\int_{\Omega} u(\omega) v(\omega) d \lambda \text { for } u \in E \text { and }\left\|F_{v}\right\|=\|v\|_{E^{\prime}}
$$

(see [13, Theorem 6.1.1]). The Mackey topology $\tau\left(E, E^{\prime}\right)$ is a locally convexsolid Hausdorff topology with the Lebesgue property (see [9, Corollary 82H]). Then $\tau\left(E, E^{\prime}\right) \subset \mathcal{T}_{E}$ and $\tau\left(E, E^{\prime}\right)=\mathcal{T}_{E}$ if the norm $\|\cdot\|_{E}$ is order continuous.

The most important classes of Banach function spaces are Lebesgue spaces $L^{p}$ $(1 \leq p \leq \infty)$ and Orlicz spaces $L^{\varphi}$ (see [19]).

Now we present a characterization of $\left(\tau\left(E, E^{\prime}\right),\|\cdot\|_{X}\right)$-continuous linear operators $T: E \rightarrow X$ (see [17, Proposition 2.2]).

Proposition 1.1 For a bounded linear operator $T: E \rightarrow X$ the following statements are equivalent:

(i) $T$ is $\left(\tau\left(E, E^{\prime}\right),\|\cdot\|_{X}\right)$-continuous.

(ii) $\left\|T\left(u_{n}\right)\right\|_{X} \rightarrow 0$ if $u_{n}(\omega) \rightarrow 0 \lambda$-a.e. and $\left|u_{n}(\omega)\right| \leq|u(\omega)| \lambda$-a.e. for some $u \in E$ and all $n \in \mathbb{N}$.

(iii) For each $u \in E,\left\|T\left(u \mathbb{1}_{A_{n}}\right)\right\|_{X} \rightarrow 0$ whenever $\lambda\left(A_{n}\right) \rightarrow 0$.

For terminology and basic facts concerning vector measure, we refer the reader to $[4,6,7,22]$. For a finitely additive measure $m: \Sigma \rightarrow X$, by $|m|(A)$ we denote the variation of $m$ on $A \in \Sigma$. A measure $m: \Sigma \rightarrow X$ is said to be $\lambda$-continuous if $\left\|m\left(A_{n}\right)\right\|_{X} \rightarrow 0$ whenever $\lambda\left(A_{n}\right) \rightarrow 0$.

Let $L^{1}(X)$ denote the Banach space of $\lambda$-equivalence classes of all $X$-valued Bochner integrable functions $g$ defined on $\Omega$, equipped with the norm $\|g\|_{1}:=$ $\int_{\Omega}\|g(\omega)\|_{X} d \lambda$.

Recall that a $\lambda$-continuous measure $m: \Sigma \rightarrow X$ of finite variation is said to have the Radon-Nikodym property with respect to $\lambda$ if there exists a function $g \in L^{1}(X)$ 
such that $m(A)=\int_{A} g(\omega) d \lambda$ for all $A \in \Sigma$. Then we write $m=g \lambda$ and a function $g$ is called the density of $m$ with respect to $\lambda$.

Assume that $m: \Sigma \rightarrow X$ is a $\lambda$-continuous measure. Following [6, $\S 13]$ for $A \in \Sigma$, we put

$$
|m|_{E^{\prime}}(A):=\sup \left\{\sum_{i=1}^{n}\left|c_{i}\right|\left\|m\left(A_{i}\right)\right\|_{X}\right\},
$$

where the supremum is taken for all functions $s=\sum_{i=1}^{n} c_{i} \mathbb{1}_{A_{i}} \in \mathcal{S}(\Sigma)$ such that $A_{i} \subset A$ for $1 \leq i \leq n$ and $\|s\|_{E} \leq 1$. The set function $|m|_{E^{\prime}}$ will be called a $E^{\prime}$-variation of the measure $m$.

If, in particular, $E=L^{\infty}$, then $|m|_{L^{1}}(A)=|m|(A)$ for $A \in \Sigma$.

Let $L^{0}(X)$ stand for the linear space of $\lambda$-equivalence classes of all strongly $\Sigma$ measurable functions $g: \Omega \rightarrow X$. Let

$$
E(X)=\left\{g \in L^{0}(X):\|g(\cdot)\|_{X} \in E\right\} .
$$

Then $E(X)$ equipped with the norm $\|g\|_{E(X)}:=\|\cdot\| g(\cdot)\left\|_{X}\right\|_{E}$ is a Banach space, called a Köthe-Bochner space (see [14]).

Definition 1.1 A bounded linear operator $T: E \rightarrow X$ is said to be Bochner representable, if there exists $g \in E^{\prime}(X)$ such that

$$
T(u)=\int_{\Omega} u(\omega) g(\omega) d \lambda \text { for } u \in E .
$$

The concept of nuclear operators between Banach spaces in due to Ruston [21]. Grothendieck carried over the concept of nuclear operators to locally convex spaces [10,11] (see also [26, p. 289], [18], [23, Chap. 3, § 7], [4, Chap. 6], [5,22]).

Following [23, Chap. 3, §7] (see also [2, Chap.4], [12, 17.3, p. 379]), we have

Definition 1.2 A linear operator $T: E \rightarrow X$ is said to be $\tau\left(E, E^{\prime}\right)$-nuclear if there exist a sequence $\left(v_{n}\right)$ in $E^{\prime}$ such that the family $\left\{F_{v_{n}}: n \in \mathbb{N}\right\}$ is $\tau\left(E, E^{\prime}\right)$ equicontinuous, a bounded sequence $\left(x_{n}\right)$ in $X$ and a sequence $\left(\alpha_{n}\right) \in \ell^{1}$ such that

$$
T(u)=\sum_{n=1}^{\infty} \alpha_{n}\left(\int_{\Omega} u v_{n} d \lambda\right) x_{n} \text { for } u \in E .
$$

Let

$$
\|T\|_{n u c}:=\inf \left\{\sum_{n=1}^{\infty}\left|\alpha_{n}\right|\left\|v_{n}\right\|_{E^{\prime}}\left\|x_{n}\right\|_{X}\right\},
$$

where the infimum is taken over all sequences $\left(v_{n}\right)$ in $E^{\prime},\left(x_{n}\right)$ in $X$ and $\left(\alpha_{n}\right) \in \ell^{1}$ such that $T$ admits a representation (1.1). 
It is known that a $\tau\left(E, E^{\prime}\right)$-nuclear operator $T: E \rightarrow X$ is $\left(\tau\left(E, E^{\prime}\right),\|\cdot\|_{X}\right)$ continuous and $\tau\left(E, E^{\prime}\right)$-compact, that is, $T(V)$ is relatively norm compact in $X$ for some $\tau\left(E, E^{\prime}\right)$-neighborhood $V$ of 0 in $E$ (see [23, Chap.3, §7, Corollary 1], [12, Corollary 4, p. 379]).

In this paper we study $\tau\left(E, E^{\prime}\right)$-nuclear operators $T: E \rightarrow X$. In Section 2 it is shown that every $\tau\left(E, E^{\prime}\right)$-nuclear operator $T: E \rightarrow X$ is Bochner representable (see Theorem 2.3 below). In particular, we obtain that a linear operator $T: L^{\infty} \rightarrow X$ is $\tau\left(L^{\infty}, L^{1}\right)$-nuclear if and only if its representing measure $m_{T}: \Sigma \rightarrow X$ has the Radon-Nikodym property and $\left|m_{T}\right|(\Omega)=\|T\|_{n u c}$ (see Theorem 2.5 below). As an application, we obtain that some natural kernel operators on $L^{\infty}$ are $\tau\left(L^{\infty}, L^{1}\right)$ nuclear (see Proposition 2.9 below). In Section 3 it is shown that every $\tau\left(L^{\infty}, L^{1}\right)$ nuclear operator $T: L^{\infty} \rightarrow X$ admits a factorization through some Orlicz space $L^{\varphi}$, that is, $T=S \circ i_{\infty}$, where $S: L^{\varphi} \rightarrow X$ is a Bochner representable, compact operator and $i_{\infty}: L^{\infty} \rightarrow L^{\varphi}$ denotes the inclusion map (see Corollary 3.5).

\section{Nuclear operators on Banach function spaces}

Assume that $T: E \rightarrow X$ is a linear operator. Then the measure $m_{T}: \Sigma \rightarrow X$ defined by

$$
m_{T}(A):=T\left(\mathbb{1}_{A}\right) \text { for } A \in \Sigma
$$

is called a representing measure of $T$.

If, in particular, $T$ is $\left(\tau\left(E, E^{\prime}\right),\|\cdot\|_{X}\right)$-continuous, then using Proposition 1.1 we obtain that $m_{T}$ is countably additive. Since $m_{T}(A)=0$ if $\lambda(A)=0$, by the Pettis theorem $m_{T}$ is $\lambda$-continuous, that is, $m_{T} \ll \lambda$.

The following lemma will be useful.

Lemma 2.1 Let $T: E \rightarrow X$ be a $\left(\tau\left(E, E^{\prime}\right),\|\cdot\|_{X}\right)$-continuous linear operator. If $\left|m_{T}\right|_{E^{\prime}}(\Omega)<\infty$ and $m_{T}$ has the Radon-Nikodym property with respect to $\lambda$ with a density $g \in L^{1}(X)$, then $g \in E^{\prime}(X)$ and

$$
\left\|\mathbb{1}_{A} g\right\|_{E^{\prime}(X)}=\left|m_{T}\right|_{E^{\prime}}(A) \text { for all } A \in \Sigma,
$$

and

$$
T(u)=\int_{\Omega} u(\omega) g(\omega) d \lambda \text { for all } u \in E
$$

Proof First we shall show that for $A \in \Sigma$,

$$
\left|m_{T}\right|_{E^{\prime}}(A)=\sup \left\{\int_{A}|s(\omega)|\|g(\omega)\|_{X} d \lambda: s \in \mathcal{S}(\Sigma),\|s\|_{E} \leq 1\right\} .
$$


Note that $\left|m_{T}\right|(A)=\int_{A}\|g(\omega)\|_{X} d \lambda$. For $s=\sum_{i=1}^{k} c_{i} \mathbb{1}_{A_{i}} \in \mathcal{S}(\Sigma)$, we have

$$
\sum_{i=1}^{k} c_{i} m_{T}\left(A \cap A_{i}\right)=\sum_{i=1}^{k} c_{i} \int_{A \cap A_{i}} g(\omega) d \lambda=\int_{A} s(\omega) g(\omega) d \lambda
$$

We now show that for $s=\sum_{i=1}^{k} c_{i} \mathbb{1}_{A_{i}} \in \mathcal{S}(\Sigma)$ and $\|s\|_{E} \leq 1$, we have

$$
\sum_{i=1}^{k}\left|c_{i}\right| \int_{A \cap A_{i}}\|g(\omega)\|_{X} d \lambda=\sum_{i=1}^{k}\left|c_{i}\right|\left|m_{T}\right|\left(A \cap A_{i}\right) \leq\left|m_{T}\right|_{E^{\prime}}(A) .
$$

Indeed, let $\varepsilon>0$ be given. Then for each $1 \leq i \leq k$, there exists a $\Sigma$-partition $\left(A_{i, j}\right)_{j=1}^{j_{i}}$ of $A \cap A_{i}$ such that

$$
\left|m_{T}\right|\left(A \cap A_{i}\right) \leq \sum_{j=1}^{j_{i}}\left\|m_{T}\left(A_{i j}\right)\right\|_{X}+\frac{\varepsilon}{k\left|c_{i}\right|}
$$

Hence

$$
\sum_{i=1}^{k}\left|c_{i}\right|\left|m_{T}\right|\left(A \cap A_{i}\right) \leq \sum_{i=1}^{k}\left(\sum_{j=1}^{j_{i}}\left\|c_{i} m_{T}\left(A_{i j}\right)\right\|_{X}\right)+\varepsilon \leq\left|m_{T}\right|_{E^{\prime}}(A)+\varepsilon
$$

because $\sum_{i=1}^{k}\left(\sum_{j=1}^{j_{i}} c_{i} \mathbb{1}_{A_{i j}}\right)=\sum_{i=1}^{k} c_{i} \mathbb{1}_{A_{i}}$.

Then we have

$$
\begin{aligned}
& \sum_{i=1}^{k}\left|c_{i}\right|\left\|m_{T}\left(A \cap A_{i}\right)\right\|_{X} \leq \sum_{i=1}^{k}\left|c_{i}\right|\left|m_{T}\right|\left(A \cap A_{i}\right) \\
& =\sum_{i=1}^{k}\left|c_{i}\right| \int_{A \cap A_{i}}\|g(\omega)\|_{X} d \lambda=\int_{A}\left(\sum_{i=1}^{k}\left|c_{i}\right| \mathbb{1}_{A_{i}}(\omega)\right)\|g(\omega)\|_{X} d \lambda \\
& =\int_{A}|s(\omega)|\|g(\omega)\|_{X} d \lambda \leq\left|m_{T}\right|_{E^{\prime}}(A) .
\end{aligned}
$$

Taking supremum on the left side, we get

$$
\left|m_{T}\right|_{E^{\prime}}(A)=\sup \left\{\int_{A}|s(\omega)|\|g(\omega)\|_{X} d \lambda: s \in \mathcal{S}(\Sigma),\|s\|_{E} \leq 1\right\} .
$$

We shall now show that $g \in E^{\prime}(X)$, that is, $\|g(\cdot)\|_{X} \in E^{\prime}$.

Indeed, let $u \in E$. Then there exists a sequence $\left(s_{n}\right)$ in $\mathcal{S}(\Sigma)$ such that $0 \leq s_{n}(\omega) \uparrow$ $|u(\omega)| \lambda$-a.e. (see [13, Corollary I.6]). Choose $c>0$ such that $\|c u\|_{E} \leq 1$. Then by the Fatou lemma, 


$$
\int_{\Omega} c|u(\omega)|\|g(\omega)\|_{X} d \lambda \leq \sup _{n} \int_{\Omega} c s_{n}(\omega)\|g(\omega)\|_{X} d \lambda \leq\left|m_{T}\right|_{E^{\prime}}(\Omega),
$$

and this means that $\|g(\cdot)\|_{X} \in E^{\prime}$, that is, $g \in E^{\prime}(X)$. Moreover, for $A \in \Sigma$ and $u \in E$ with $\|u\|_{E} \leq 1$, using (2.1) we get

$$
\int_{\Omega}|u(\omega)|\left\|\mathbb{1}_{A}(\omega) g(\omega)\right\|_{X} d \lambda \leq \sup _{n} \int_{\Omega} s_{n}(\omega)\left\|\mathbb{1}_{A}(\omega) g(\omega)\right\|_{X} d \lambda \leq\left|m_{T}\right|_{E^{\prime}}(A)
$$

and it follows that $\left\|\mathbb{1}_{A} g\right\|_{E^{\prime}(X)} \leq\left|m_{T}\right|_{E^{\prime}}(A)$. In view of (2.1) we get $\left|m_{T}\right|_{E^{\prime}}(A) \leq$ $\left\|\mathbb{1}_{A} g\right\|_{E^{\prime}(X)}$. Hence $\left|m_{T}\right|_{E^{\prime}}(A)=\left\|\mathbb{1}_{A} g\right\|_{E^{\prime}(X)}$.

Note that for $s=\sum_{i=1}^{k} c_{i} \mathbb{1}_{A_{i}} \in \mathcal{S}(\Sigma)$, we have

$$
T(s)=\sum_{i=1}^{k} c_{i} m_{T}\left(A_{i}\right)=\int_{\Omega} s(\omega) g(\omega) d \lambda
$$

Let $u \in E$ be given. Then there exists a sequence $\left(s_{n}\right)$ in $\mathcal{S}(\Sigma)$ such that $\mid s_{n}(\omega)-$ $u(\omega) \mid \rightarrow 0 \lambda$-a.e. and $\left|s_{n}(\omega)\right| \leq|u(\omega)| \lambda$-a.e. for all $n \in \mathbb{N}$. Then $\left|s_{n}(\omega)-u(\omega)\right|$ $\|g(\omega)\|_{X} \leq 2|u(\omega)|\|g(\omega)\|_{X} \lambda$-a.e., where $u\|g(\cdot)\|_{X} \in L^{1}$. Hence by the Lebesgue dominated convergence theorem,

$\left\|\int_{\Omega} s_{n}(\omega) g(\omega) d \lambda-\int_{\Omega} u(\omega) g(\omega) d \lambda\right\|_{X} \leq \int_{\Omega}\left|s_{n}(\omega)-u(\omega)\right|\|g(\omega)\|_{X} d \lambda \rightarrow 0$.

On the other hand, in view of Proposition 1.1 we have

$$
\left\|T\left(s_{n}\right)-T(u)\right\|_{X}=\left\|T\left(s_{n}-u\right)\right\|_{X} \rightarrow 0
$$

Hence $T(u)=\int_{\Omega} u(\omega) g(\omega) d \lambda$.

As a consequence of Lemma 2.1, we have

Proposition 2.2 Assume that $T: E \rightarrow X$ is a Bochner representable operator, that is, there exists $g \in E^{\prime}(X)$ such that

$$
T(u)=\int_{\Omega} u(\omega) g(\omega) d \lambda \text { for all } u \in E .
$$

Then the following statements hold:

(i) $T$ is $\left(\tau\left(E, E^{\prime}\right),\|\cdot\|_{X}\right)$-continuous.

(ii) For every $A \in \Sigma,\left|m_{T}\right|_{E^{\prime}}(A)=\left\|\mathbb{1}_{A} g\right\|_{E^{\prime}(X)}$.

Proof (i) Assume that $u_{n}(\omega) \rightarrow 0 \lambda$-a.e. and $\left|u_{n}(\omega)\right| \leq|u(\omega)| \lambda$-a.e. for some $u \in E$ and all $n \in \mathbb{N}$. Since for $n \in \mathbb{N}$,

$$
\left\|T\left(u_{n}\right)\right\|_{X} \leq \int_{\Omega}\left|u_{n}(\omega)\right|\|g(\omega)\|_{X} d \lambda,
$$


where $u\|g(\cdot)\|_{X} \in L^{1}$, by the Lebesgue dominated convergence theorem, we get $\left\|T\left(u_{n}\right)\right\|_{X} \rightarrow 0$. Hence in view of Proposition 1.1 $T$ is $\left(\tau\left(E, E^{\prime}\right)\right.$, $\left.\|\cdot\|_{X}\right)$ continuous.

(ii) Assume that $s=\sum_{i=1}^{k} c_{i} \mathbb{1}_{A_{i}} \in \mathcal{S}(\Sigma)$ and $\|s\|_{E} \leq 1$. Then

$$
\begin{aligned}
\sum_{i=1}^{k}\left|c_{i}\right|\left\|m_{T}\left(A_{i}\right)\right\|_{X} & =\sum_{\substack{i=1\\
}}^{k}\left|c_{i}\right| \int_{A_{i}}\|g(\omega)\|_{X} d \lambda=\int_{\Omega}|s(\omega)|\|g(\omega)\|_{X} d \lambda \\
& \leq\|s\|_{E}\|g\|_{E^{\prime}(X)} \leq\|g\|_{E^{\prime}(X)} .
\end{aligned}
$$

Hence $\left|m_{T}\right|_{E^{\prime}}(\Omega) \leq\|g\|_{E^{\prime}(X)}$. Using (i) and Lemma 2.1, we get $\left|m_{T}\right|_{e^{\prime}}(A)=$ $\left\|\mathbb{1}_{A} g\right\|_{E^{\prime}(X)}$ for all $A \in \Sigma$.

The following result shows a relationship between $\tau\left(E, E^{\prime}\right)$-nuclear operators and Bochner representable operators $T: E \rightarrow X$.

Theorem 2.3 Assume that $T: E \rightarrow X$ is a $\tau\left(E, E^{\prime}\right)$-nuclear operator. Then $T$ is Bochner representable and $\left|m_{T}\right|_{E^{\prime}}(\Omega) \leq\|T\|_{n u c}$.

Proof Let $\varepsilon>0$ be given. Then there exists a bounded sequence $\left(v_{n}\right)$ in $E^{\prime}$, a bounded sequence $\left(x_{n}\right)$ in $X$ and a sequence $\left(\alpha_{n}\right) \in \ell^{1}$ such that

$$
T(u)=\sum_{n=1}^{\infty} \alpha_{n}\left(\int_{\Omega} u v_{n} d \lambda\right) x_{n} \text { for } u \in E
$$

and

$$
\sum_{n=1}^{\infty}\left|\alpha_{n}\right|\left\|v_{n}\right\|_{E^{\prime}}\left\|x_{n}\right\|_{X} \leq\|T\|_{n u c}+\varepsilon
$$

Hence we have

$$
m_{T}(A)=\sum_{n=1}^{\infty} \alpha_{n}\left(\int_{A} v_{n} d \lambda\right) x_{n}
$$

We shall now show that $\left|m_{T}\right|_{E^{\prime}}(\Omega) \leq\|T\|_{n u c}$. Indeed, let $s=\sum_{i=1}^{k} c_{i} \mathbb{1}_{A_{i}} \in \mathcal{S}(\Sigma)$ with $\|s\|_{E} \leq 1$. Then using (2.2) we get

$$
\begin{aligned}
\sum_{i=1}^{k}\left|c_{i}\right|\left\|m_{T}\left(A_{i}\right)\right\|_{X} & =\sum_{i=1}^{k}\left\|c_{i} \sum_{n=1}^{\infty} \alpha_{n}\left(\int_{A_{i}} v_{n}(\omega) d \lambda\right) x_{n}\right\|_{X} \\
& \leq \sum_{n=1}^{\infty}\left|\alpha_{n}\right|\left(\sum_{i=1}^{k}\left|c_{i}\right| \int_{A_{i}}\left|v_{n}(\omega)\right| d \lambda\right)\left\|x_{n}\right\|_{X} \\
& =\sum_{n=1}^{\infty}\left|\alpha_{n}\right|\left\|x_{n}\right\|_{X} \int_{\Omega}|s(\omega)|\left|v_{n}(\omega)\right| d \lambda
\end{aligned}
$$




$$
\leq \sum_{n=1}^{\infty}\left|\alpha_{n}\right|\left\|x_{n}\right\|_{X}\left\|v_{n}\right\|_{E^{\prime}} \leq\|T\|_{n u c}+\varepsilon
$$

Hence we get

$$
\left|m_{T}\right|_{E^{\prime}}(\Omega) \leq\|T\|_{n u c} .
$$

For $n \in \mathbb{N}$, let $g_{n}:=\sum_{i=1}^{n} \alpha_{i} v_{i} \otimes x_{i}$. Then for $n, k \in \mathbb{N}$ with $n>k$, we have

$$
\begin{aligned}
\int_{\Omega} \| g_{n}(\omega) & -g_{k}(\omega)\left\|_{X} d \lambda=\int_{\Omega}\right\| \sum_{i=k+1}^{n} \alpha_{i} v_{i}(\omega) x_{i} \|_{X} d \lambda \\
& \leq \int_{\Omega}\left(\sum_{i=k+1}^{n}\left|\alpha_{i}\right|\left|v_{i}(\omega)\right|\left\|x_{i}\right\|_{X}\right) d \lambda=\sum_{i=k+1}^{n}\left|\alpha_{i}\right|\left\|x_{i}\right\|_{X} \int_{\Omega}\left|v_{i}\right| d \lambda \\
& \leq \sum_{i=k+1}^{n}\left|\alpha_{i}\right|\left\|x_{i}\right\|_{X}\left\|\mathbb{1}_{\Omega}\right\|_{E}\left\|v_{i}\right\|_{E^{\prime}} \leq \sup _{j \in \mathbb{N}}\left\|x_{j}\right\|_{X} \sup _{j \in \mathbb{N}}\left\|v_{j}\right\|_{E^{\prime}}\left\|\mathbb{1}_{\Omega}\right\|_{E} \sum_{i=k+1}^{n}\left|\alpha_{i}\right| .
\end{aligned}
$$

This follows that $\left(g_{n}\right)$ is a Cauchy sequence in the Banach space $L^{1}(X)$, so there exists $g \in L^{1}(X)$ such that $\left\|g_{n}-g\right\|_{1} \rightarrow 0$. One can easily show that

$$
\left(\int_{A} v d \lambda\right) x=\int_{A} v(\omega) x d \lambda \text { for } v \in E^{\prime}, x \in X, A \in \Sigma .
$$

Hence for $A \in \Sigma$, we have

$$
\begin{aligned}
\| & \sum_{i=1}^{n} \alpha_{i}\left(\int_{A} v_{i} d \lambda\right) x_{i}-\int_{A} g d \lambda\left\|_{X}=\right\| \int_{A} \sum_{i=1}^{n} \alpha_{i} v_{i}(\omega) x_{i} d \lambda-\int_{A} g(\omega) d \lambda \|_{X} \\
\leq \int_{A}\left\|\sum_{i=1}^{n} \alpha_{i} v_{i}(\omega) x_{i}-g(\omega)\right\|_{X} d \lambda & =\int_{A}\left\|g_{n}(\omega)-g(\omega)\right\|_{X} d \lambda \rightarrow 0 .
\end{aligned}
$$

Then in view of (2.3) for $A \in \Sigma$, we get

$$
m_{T}(A)=\int_{A} g(\omega) d \lambda
$$

Using Lemma 2.1 and (2.4) we see that $g \in E^{\prime}(X)$ with $\|g\|_{E^{\prime}(X)}=\left|m_{T}\right|_{E^{\prime}}(\Omega) \leq$ $\|T\|_{n u c}$ and $T(u)=\int_{\Omega} u(\omega) g(\omega) d \lambda$ for all $u \in E$.

Now we shall study $\tau\left(L^{\infty}, L^{1}\right)$-nuclear operators $T: L^{\infty} \rightarrow X$.

Making use of the Dunford-Pettis theorem (see [3, Theorem, p. 93]) we have

Theorem 2.4 For a subset $H$ of $L^{1}$ the following statements are equivalent:

(i) $H$ is relatively weakly compact.

(ii) $\sup _{v \in H}\|v\|_{1}<\infty$ and $H$ is uniformly integrable.

(iii) $\left\{F_{v}: v \in H\right\}$ is $\tau\left(L^{\infty}, L^{1}\right)$-equicontinuous. 
Note that in view of Theorem 2.4 and Definition 1.2, a linear operator $T: L^{\infty} \rightarrow X$ is $\tau\left(L^{\infty}, L^{1}\right)$-nuclear if there exist a bounded uniformly integrable sequence $\left(v_{n}\right)$ in $L^{1}$, a bounded sequence $\left(x_{n}\right)$ in $X$ and a sequence $\left(\alpha_{n}\right) \in \ell^{1}$ such that

$$
T(u)=\sum_{n=1}^{\infty} \alpha_{n}\left(\int_{\Omega} u v_{n} d \lambda\right) x_{n} \text { for all } u \in L^{\infty}
$$

and then

$$
\|T\|_{n u c}=\inf \left\{\sum_{n=1}^{\infty}\left|\alpha_{n}\right|\left\|v_{n}\right\|_{1}\left\|x_{n}\right\|_{X}\right\}
$$

where the infimum is taken over all sequences $\left(v_{n}\right)$ in $L^{1},\left(x_{n}\right)$ in $X$ and $\left(\alpha_{n}\right) \in \ell^{1}$ such that $T$ admits a representation (2.5).

Now we can state our main result.

Theorem 2.5 For a linear operator $T: L^{\infty} \rightarrow X$ the following statements are equivalent:

(i) $m_{T}$ has the Radon-Nikodym property with respect to $\lambda$.

(ii) $T$ is Bochner representable.

(iii) $T$ is a $\tau\left(L^{\infty}, L^{1}\right)$-nuclear operator.

In this case $\left|m_{T}\right|(\Omega)=\|T\|_{n u c}$.

Proof (i) $\Rightarrow$ (ii) Assume that (i) holds with the density $g \in L^{1}(X)$. Note that for $s \in \mathcal{S}(\Sigma)$, we have

$$
\int_{\Omega} s d m_{T}=\int_{\Omega} s(\omega) g(\omega) d \lambda
$$

Let $u \in L^{\infty}$. Choose a sequence $\left(s_{n}\right)$ in $\mathcal{S}(\Sigma)$ such that $\left\|u-s_{n}\right\|_{\infty} \rightarrow 0$. Then we have

$$
T(u)=\int_{\Omega} u d m_{T}=\lim \int_{\Omega} s_{n} d m_{T}=\lim \int_{\Omega} s_{n}(\omega) g(\omega) d \lambda=\int_{\Omega} u(\omega) g(\omega) d \lambda .
$$

(ii) $\Rightarrow$ (i) This is obvious.

(ii) $\Rightarrow$ (iii) Assume that (ii) holds, that is, there exists $g \in L^{1}(X)$ such that

$$
T(u)=\int_{\Omega} u(\omega) g(\omega) d \lambda \text { for all } u \in L^{\infty} .
$$

Let $L^{1} \hat{\otimes} X$ denote the projective tensor product of $L^{1}$ and $X$, equipped with the norm $\pi$ defined for $w \in L^{1} \hat{\otimes} X$, by

$$
\pi(w):=\inf \left\{\sum_{n=1}^{\infty}\left|\alpha_{n}\right|\left\|v_{n}\right\|_{1}\left\|x_{n}\right\|_{X}\right\}
$$


where the infimum is taken over all sequences $\left(v_{n}\right)$ in $L^{1},\left(x_{n}\right)$ in $X$ with $\lim \left\|v_{n}\right\|_{1}=$ $0=\lim \left\|x_{n}\right\|_{X}$ and $\left(\alpha_{n}\right) \in \ell^{1}$ such that $w=\sum_{n=1}^{\infty} \alpha_{n} v_{n} \otimes x_{n}$ (see [22, Proposition 2.8, pp. 21-22]). It is known that $L^{1} \hat{\otimes} X$ is isometrically isomorphic to $L^{1}(X)$ through the isometry $J$, where

$$
J(v \otimes x):=v(\cdot) x \text { for } v \in L^{1}, x \in X
$$

(see [4, Example 10, p. 228], [22, Example 2.19, p. 29], [5, Theorem 1.1.10, p. 14]). Let $\varepsilon>0$ be given. Then there exist sequence $\left(v_{n}\right)$ in $L^{1}$ and $\left(x_{n}\right)$ in $X$ with $\lim _{n}\left\|v_{n}\right\|_{1}=$ $0=\lim \left\|x_{n}\right\|_{X}$ and $\left(\alpha_{n}\right) \in \ell^{1}$ such that

$$
J^{-1}(g)=\sum_{n=1}^{\infty} \alpha_{n} v_{n} \otimes x_{n} \text { in }\left(L^{1} \hat{\otimes} X, \pi\right)
$$

and

$$
\sum_{n=1}^{\infty}\left|\alpha_{n}\right|\left\|v_{n}\right\|_{1}\left\|x_{n}\right\|_{X} \leq \pi\left(J^{-1}(g)\right)+\varepsilon=\|g\|_{1}+\varepsilon .
$$

Hence

$$
g=J\left(\sum_{n=1}^{\infty} \alpha_{n} v_{n} \otimes x_{n}\right)=\sum_{n=1}^{\infty} \alpha_{n} v_{n}(\cdot) x_{n} \text { in }\left(L^{1}(X),\|\cdot\|_{1}\right)
$$

Note that

$$
\begin{aligned}
& \left\|\int_{\Omega} u(\omega) g(\omega) d \lambda-\sum_{i=1}^{n} \alpha_{i}\left(\int_{\Omega} u(\omega) v_{i}(\omega) d \lambda\right) x_{i}\right\|_{X} \\
& \leq \int_{\Omega}|u(\omega)|\left\|g(\omega)-\sum_{i=1}^{n} \alpha_{i} v_{i}(\omega) x_{i}\right\|_{X} d \lambda \\
& \quad \leq\|u\|_{\infty} \int_{\Omega}\left\|g(\omega)-\sum_{i=1}^{n} \alpha_{i} v_{i}(\omega) x_{i}\right\|_{X} d \lambda=\|u\|_{\infty} \cdot\left\|g-\sum_{i=1}^{n} \alpha_{i} v_{i} \otimes x_{i}\right\|_{1}
\end{aligned}
$$

Hence

$$
T(u)=\sum_{n=1}^{\infty} \alpha_{n}\left(\int_{\Omega} u v_{n} d \lambda\right) x_{n} \text { for } u \in L^{\infty}
$$

Since $\lim \left\|v_{n}\right\|_{1}=0$, the set $\left\{v_{n}: n \in \mathbb{N}\right\}$ is uniformly integrable and it follows that $T$ is $\tau\left(L^{\infty}, L^{1}\right)$-nuclear. In view of (2.6) we get

$$
\|T\|_{n u c} \leq\|g\|_{1}=\left|m_{T}\right|(\Omega)
$$


(iii) $\Rightarrow$ (ii) Assume that (iii) holds. Then by Theorem $2.3 T$ is Bochner representable and

$$
\left|m_{T}\right|(\Omega)=\|g\|_{1} \leq\|T\|_{n u c} .
$$

Thus (i) $\Leftrightarrow\left(\right.$ ii) $\Leftrightarrow$ (iii) hold and using (2.7) and (2.8), we get $\left|m_{T}\right|(\Omega)=\|T\|_{n u c}$.

Let $b a_{\lambda}(\Sigma)$ denote the Banach space of all bounded finitely additive real measures $\mu$ on $\Sigma$ such that $\mu(A)=0$ if $\lambda(A)=0$, equipped with norm $\|\mu\|:=|\mu|(\Omega)$. The Banach dual $\left(L^{\infty}\right)^{*}$ of $L^{\infty}$ can be identified with $b a_{\lambda}(\Sigma)$ through the integration mapping $b a_{\lambda}(\Sigma) \ni \mu \mapsto F_{\mu} \in\left(L^{\infty}\right)^{*}$, where $F_{\mu}(u)=\int_{\Omega} u d \mu$ for all $u \in L^{\infty}$ and $|\mu|(\Omega)=\left\|F_{\mu}\right\|$.

Recall (see [26, p. 279]) that a linear operator $T: L^{\infty} \rightarrow X$ is said to be nuclear if there exist a bounded sequence $\left(\mu_{n}\right)$ in $b a_{\lambda}(\Sigma)$, a bounded sequence $\left(x_{n}\right)$ in $X$ and a sequence $\left(\alpha_{n}\right) \in \ell^{1}$ such that

$$
T(u)=\sum_{n=1}^{\infty} \alpha_{n}\left(\int_{\Omega} u d \mu_{n}\right) x_{n} \text { for all } u \in L^{\infty} .
$$

It is well known that a bounded linear operator $T: L^{\infty} \rightarrow X$ is $\left(\tau\left(L^{\infty}, L^{1}\right),\|\cdot\|_{X}\right)$ continuous if and only if its representing measure $m_{T}: \Sigma \rightarrow X$ is $\lambda$-continuous (see [17, Proposition 3.1]). Hence due to Swartz (see [24, Theorem 1 and Theorem 3]) we have

Theorem 2.6 Assume that $T: L^{\infty} \rightarrow X$ is a $\left(\tau\left(L^{\infty}, L^{1}\right),\|\cdot\|_{X}\right)$-continuous linear operator. Then the following statements are equivalent:

(i) $T$ is nuclear.

(ii) $m_{T}$ has the Radon-Nikodym property with respect to $\lambda$.

Combining Theorem 2.5 and Theorem 2.6, we get

Corollary 2.7 For a linear operator $T: L^{\infty} \rightarrow X$ the following statements are equivalent:

(i) $T$ is $\tau\left(L^{\infty}, L^{1}\right)$-nuclear.

(ii) $T$ is $\left(\tau\left(L^{\infty}, L^{1}\right),\|\cdot\|_{X}\right)$-continuous and nuclear.

As an application of Theorem 2.5 we show that some natural kernel operators on $L^{\infty}$ are $\tau\left(L^{\infty}, L^{1}\right)$-nuclear.

Assume that $K_{1}$ and $K_{2}$ are compact Hausdorff spaces and $k(\cdot, \cdot) \in C\left(K_{2} \times K_{1}\right)$. Let $\mathcal{B} o$ be the $\sigma$-algebra of Borel sets in $K_{1}$ and $\lambda: \mathcal{B} o \rightarrow[0, \infty)$ be a countably additive measure.

We will need the following lemma.

Lemma 2.8 For every $u \in L^{\infty}(\lambda)$, the mapping $\Psi_{u}: K_{1} \ni s \mapsto u(s) k(\cdot, s) \in C\left(K_{2}\right)$ is continuous. 
Proof Let $s_{0} \in K_{1}$ and $\varepsilon>0$ be given. Then for every $t \in K_{2}$ there exist a neighborhood $V_{t}$ of $t$ and a neighborhood $W_{t}$ of $s_{0}$ such that

$$
\left|k(z, s)-k\left(t, s_{0}\right)\right| \leq \frac{\varepsilon}{\|u\|_{\infty}} \text { for all } z \in V_{t}, s \in W_{t} .
$$

Hence there exist $t_{1}, \ldots, t_{n} \in K_{2}$ such that $K_{2}=\bigcup_{i=1}^{n} V_{t_{i}}$. Let us put $W:=$ $\bigcap_{i=1}^{n} W_{t_{i}}$. For $t \in K_{2}$, choose $i_{0}$ with $1 \leq i_{0} \leq n$ such that $t \in V_{t_{i}}$. Then for $s \in W$, we have $\mid k(t, s)-k\left(t, s_{0} \mid \leq \frac{\varepsilon}{\|u\|_{\infty}}\right.$. Hence

$$
\left\|\Psi_{u}(s)-\Psi_{u}\left(s_{0}\right)\right\|_{\infty}=\sup _{t \in K_{2}}\left|k(t, s)-k\left(t, s_{0}\right)\right|\|u\|_{\infty} \leq \varepsilon .
$$

This means that $\Psi_{u}$ is continuous.

Note that the Banach space $C\left(K_{1}, C\left(K_{2}\right)\right)$ can be embedded in the Banach space $L^{1}\left(C\left(K_{2}\right)\right)$ such that with each function from $C\left(K_{1}, C\left(K_{2}\right)\right)$ is associated its $\lambda$ equivalence class in $L^{1}\left(C\left(K_{2}\right)\right)$.

In view of Lemma 2.8 we can define a kernel operator $T_{k}: L^{\infty}(\lambda) \rightarrow C\left(K_{2}\right)$ by

$$
T_{k}(u):=\int_{K_{1}} u(s) k(\cdot, s) d \lambda(s) \text { for all } u \in L^{\infty}(\lambda)
$$

For $t \in K_{2}$, let $\delta_{t}(w):=w(t)$ for all $w \in C\left(K_{2}\right)$. Then $\delta_{t} \in C\left(K_{2}\right)^{*}$ and according to the Hille's theorem (see [DU, Theorem 6, p. 47]), we have

$$
T_{k}(u)(t)=\delta_{t}\left(T_{k}(u)\right)=\int_{K_{1}} u(s) k(t, s) d \lambda(s) \text { for all } u \in L^{\infty}(\lambda), t \in K_{2} .
$$

Then

$$
m_{T_{k}}(A)=T_{k}\left(\mathbb{1}_{A}\right)=\int_{A} k(\cdot, s) d \lambda(s) \text { for all } A \in \mathcal{B} o,
$$

where the mapping $K_{1} \ni s \mapsto k(\cdot, s) \in C\left(K_{2}\right)$ belongs to $L^{1}\left(C\left(K_{2}\right)\right)$.

Hence for $A \in \mathcal{B} o$,

$$
\left|m_{T_{k}}\right|(A)=\int_{A}\|k(\cdot, s)\|_{\infty} d \lambda(s)=\int_{A} \sup _{t \in K_{2}}|k(t, s)| d \lambda(s) .
$$

Hence as a consequence of Theorem 2.5 we have

Proposition 2.9 The kernel operator $T_{k}: L^{\infty}(\lambda) \rightarrow C\left(K_{2}\right)$ is $\tau\left(L^{\infty}(\lambda), L^{1}(\lambda)\right)$ nuclear and

$$
\left\|T_{k}\right\|_{n u c}=\int_{K_{1}} \sup _{t \in K_{2}}|k(t, s)| d \lambda(s) .
$$




\section{Application of the theory of Orlicz spaces to vector measures}

First we recall terminology and basic facts concerning Orlicz spaces and OrliczBochner spaces (see [19] for more details). By a Young function we mean here a convex continuous mapping $\varphi:[0, \infty) \rightarrow[0, \infty)$ that vanishes only at 0 and $\varphi(t) / t$ $\rightarrow 0$ as $t \rightarrow 0$ and $\varphi(t) / t \rightarrow \infty$ as $t \rightarrow \infty$. By $\varphi^{*}$ we denote the complementary function of $\varphi$ in the sense of Young, that is, $\varphi^{*}(t)=\sup \{t s-\varphi(s): s \geq 0\}$ for $t \geq 0$. Note that $\varphi^{* *}=\varphi$.

The corresponding Orlicz space $L^{\varphi}$ is an ideal of $L^{0}$ defined by

$$
L^{\varphi}:=\left\{u \in L^{0}: \int_{\Omega} \varphi(\alpha|u(\omega)|) d \lambda<\infty \text { for some } \alpha>0\right\},
$$

and equipped with the topology $\mathcal{T}_{\varphi}$, defined by two equivalent norms:

$$
\begin{aligned}
& \|u\|_{\varphi}:=\inf \left\{\alpha>0: \int_{\Omega} \varphi(|u(\omega)| / \alpha) d \lambda \leq 1\right\}, \\
& \|u\|_{\varphi}^{0}:=\sup \left\{\int_{\Omega}|u(\omega) v(\omega)| d \lambda: v \in L^{\varphi^{*}}, \int_{\Omega} \varphi^{*}(|v(\omega)|) d \lambda \leq 1\right\},
\end{aligned}
$$

called the Luxemburg norm and the Orlicz norm. Then we have:

$\|u\|_{\varphi} \leq 1$ if and only if $\int_{\Omega} \varphi(|u(\omega)|) d \lambda \leq 1$,

$\left\|u_{n}\right\|_{\varphi} \rightarrow 0$ if and only if $\int_{\Omega} \varphi\left(\alpha\left|u_{n}(\omega)\right|\right) d \lambda \rightarrow 0$ for every $\alpha>0$,

$$
\begin{aligned}
E^{\varphi} & :=\left\{u \in L^{\varphi}: \int_{\Omega} \varphi(\alpha|u(\omega)|) d \lambda<\infty \text { for all } \alpha>0\right\} \\
& =\left\{u \in L^{\varphi}:\left\|\mathbb{1}_{A_{n}} u\right\|_{\varphi} \rightarrow 0 \text { if } \lambda\left(A_{n}\right) \rightarrow 0\right\}
\end{aligned}
$$

$\left(L^{\varphi}\right)^{\prime}=L^{\varphi^{*}}$.

The Orlicz-Bochner space $L^{\varphi}(X)$ is defined by

$$
\begin{aligned}
L^{\varphi}(X) & :=\left\{g \in L^{0}(X): \int_{\Omega} \varphi\left(\alpha\|g(\omega)\|_{X}\right) d \lambda<\infty \text { for some } \alpha>0\right\} \\
& =\left\{g \in L^{0}(X):\|g(\cdot)\|_{X} \in L^{\varphi}\right\} .
\end{aligned}
$$

For $g \in L^{\varphi}(X)$, let

$$
\|g\|_{\varphi}:=\|\| g(\cdot)\left\|_{X}\right\|_{\varphi} \text { and }\|g\|_{\varphi}^{0}:=\|\| g(\cdot)\left\|_{X}\right\|_{\varphi}^{0} .
$$

Then

$$
E^{\varphi}(X):=\left\{g \in L^{0}(X):\|g(\cdot)\|_{X} \in E^{\varphi}\right\}
$$

Assume that $\varphi$ is Young function. If $m: \Sigma \rightarrow X$ is a $\lambda$-continuous measure, we write $|m|_{\varphi^{*}}$ instead of $|m|_{\left(L^{\varphi}\right)^{\prime}}$. 
Lemma 3.1 Assume that $m: \Sigma \rightarrow X$ is a $\lambda$-continuous measure and $\varphi$ is Young function. Then for $A \in \Sigma$, we have

$$
|m|(A) \leq\left(\varphi^{-1}\left(\frac{1}{\lambda(A)}\right)\right)^{-1}|m|_{\varphi^{*}}(A) .
$$

Proof Let $A \in \Sigma$ and $\varepsilon>0$ be given. Then there is a $\Sigma$-partition $\left(A_{i}\right)_{i=1}^{n}$ of $A$ such that

$$
|m|(A) \leq \sum_{i=1}^{n}\left\|m\left(A_{i}\right)\right\|_{X}+\left(\varphi^{-1}\left(\frac{1}{\lambda(A)}\right)\right)^{-1} \varepsilon
$$

Hence

It is known that $\left\|\mathbb{1}_{A}\right\|_{\varphi}=\left(\varphi^{-1}\left(\frac{1}{\lambda(A)}\right)\right)^{-1}$ (see [RR, Chap. 3.4, Corollary 7, p. 79]).

$$
\left\|\sum_{i=1}^{n} \varphi^{-1}\left(\frac{1}{\lambda(A)}\right) \mathbb{1}_{A_{i}}\right\|_{\varphi}=\left\|\varphi^{-1}\left(\frac{1}{\lambda(A)}\right) \mathbb{1}_{A}\right\|_{\varphi}=1
$$

and

$$
\varphi^{-1}\left(\frac{1}{\lambda(A)}\right)|m|(A) \leq \sum_{i=1}^{n} \varphi^{-1}\left(\frac{1}{\lambda(A)}\right)\left\|m\left(A_{i}\right)\right\|_{X}+\varepsilon
$$

It follows that $\varphi^{-1}\left(\frac{1}{\lambda(A)}\right)|m|(A) \leq|m|_{\varphi^{*}}(A)$, so

$$
|m|(A) \leq \varphi^{-1}\left(\frac{1}{\lambda(A)}\right)^{-1}|m|_{\varphi^{*}}(A) .
$$

By $\gamma\left[\mathcal{T}_{\varphi},\left.\mathcal{T}_{0}\right|_{L^{\varphi}}\right]$ (in brief, $\gamma_{\varphi}$ ) we denote the natural mixed topology on $L^{\varphi}$ (in the sense of Wiweger), that is, $\gamma_{\varphi}$ is the finest linear topology that agrees with $\mathcal{T}_{0}$ on $\mathcal{T}_{\varphi}$-bounded sets in $L^{\varphi}$ (see [1,25] for more details). Then $\gamma_{\varphi}$ is a locally convexsolid Hausdorff topology and $\gamma_{\varphi}$ and $\mathcal{T}_{\varphi}$ have the same bounded sets. This means that $\left(L^{\varphi}, \gamma_{\varphi}\right)$ is a generalized DF-space (see [20]) and it follows that $\left(L^{\varphi}, \gamma_{\varphi}\right)$ is quasinormable (see [20, p. 422]). Hence as a consequence of the Grothendieck's classical result (see [20, p. 429]), we have

Proposition 3.2 For a linear operator $S: L^{\varphi} \rightarrow X$ the following statements are equivalent:

(i) $S$ is $\left(\gamma_{\varphi},\|\cdot\|_{X}\right)$-continuous and compact.

(ii) $S$ is $\gamma_{\varphi}$-compact, that is, there exists a $\gamma_{\varphi}$-neighborhood $V$ of 0 in $L^{\varphi}$ such that $T(V)$ is relatively norm compact in $X$. 
We say that a Young function $\varphi$ increases essentially more rapidly than another $\psi$ (in symbols, $\psi \ll \varphi$ ) if for arbitrary $c>0, \psi(c t) / \varphi(t) \rightarrow 0$ as $t \rightarrow 0$ and $t \rightarrow \infty$. Note that $L^{\varphi} \subset E^{\psi}$ if $\psi \ll \varphi$.

The following result will be useful (see [16, Theorem 2.1]).

Theorem 3.3 Let $\varphi$ be a Young function. Then

(i) $\gamma_{\varphi}$ is generated by the family of norms $\left\{\left.\|\cdot\|_{\psi}\right|_{L^{\varphi}}: \psi \ll \varphi\right\}$.

(ii) $\left(L^{\varphi}, \gamma_{\varphi}\right)^{*}=\left\{F_{v}: v \in E^{\varphi^{*}}\right\}$, where $F_{v}(u)=\int_{\Omega} u(\omega) v(\omega) d \lambda$ for all $u \in L^{\varphi}$.

According to [15, Corollary 1.6] we have the following identity:

$$
L^{1}=\bigcup_{\varphi} E^{\varphi} \text {, where } \varphi \text { runs over the family of all Young functions. }
$$

Now we can state the main result in this section.

Theorem 3.4 Assume that a measure $m: \Sigma \rightarrow X$ has the Radon-Nikodym property with the density $g \in L^{1}(X)$. Then there exists a Young function $\varphi$ such that $g \in E^{\varphi^{*}}(X)$ and the following statements hold:

(i) The operator $S_{g}: L^{\varphi} \rightarrow X$ defined by

$$
S_{g}(u):=\int_{\Omega} u(\omega) g(\omega) d \lambda \text { for all } u \in L^{\varphi}
$$

is $\left(\gamma_{\varphi},\|\cdot\|_{X}\right)$-continuous and compact.

(ii) $S_{g}$ is $\gamma_{\varphi}$-compact, that is, there exists a Young function $\psi$ with $\psi \ll \varphi$ such that $\left\{\int_{\Omega} u(\omega) g(\omega) d \lambda: u \in L^{\varphi},\|u\|_{\psi} \leq 1\right\}$ is a relatively norm compact subset of $X$.

(iii) $|m|_{\varphi^{*}}(\Omega)<\infty$ and $|m|_{\varphi^{*}}(A)=\left\|\mathbb{1}_{A} g\right\|_{\varphi^{*}}^{0}$ for every $A \in \Sigma$.

(iv) $|m|_{\varphi^{*}}$ is $\lambda$-continuous, that is, $|m|_{\varphi^{*}}\left(A_{n}\right) \rightarrow 0$ if $\lambda\left(A_{n}\right) \rightarrow 0$.

Proof In view of (3.1) there exists a Young function $\psi$ such that $g \in E^{\psi}(X)$, that is, $\|g(\cdot)\|_{X} \in E^{\psi}$. Hence $|u|\|g(\cdot)\|_{X} \in L^{1}$ for every $u \in L^{\psi^{*}}$. Let us put $\varphi=\psi^{*}$. Then $\varphi^{*}=\psi^{* *}=\psi$ and $\left(L^{\varphi}\right)^{\prime}=L^{\varphi^{*}}=L^{\psi}$ and $L^{\psi^{*}}=L^{\varphi}$, and so $g \in E^{\varphi^{*}}(X)$. Then for $u \in L^{\varphi}$, we have

$$
\left\|S_{g}(u)\right\|_{X} \leq \int_{\Omega}|u(\omega)|\|g(\omega)\|_{X} d \lambda=F_{\|g(\cdot)\|_{X}}(|u|)
$$

where $\|g(\cdot)\|_{X} \in E^{\varphi^{*}}$. The inequality shows that $S_{g}$ is $\left(|\sigma|\left(L^{\varphi}, E^{\varphi^{*}}\right),\|\cdot\|_{X}\right)$ continuous, where $|\sigma|$ denotes the absolute weak topology. Since $\left(|\sigma|\left(L^{\varphi}, E^{\varphi^{*}}\right)\right.$ is the coarsest locally convex-solid topology on $L^{\varphi}$ with dual $E^{\varphi^{*}}$, and by Theorem 3.3(ii) $\gamma_{\varphi}$ is such a topology, it follows that $S_{g}$ is $\left(\gamma_{\varphi},\|\cdot\|_{X}\right)$-continuous.

To show that $S_{g}$ is compact, choose a sequence $\left(f_{n}\right)$ of $X$-valued simple functions on $\Omega$ such that $\left\|f_{n}(\omega)-g(\omega)\right\|_{X} \rightarrow 0$ and $\left\|f_{n}(\omega)\right\|_{X} \leq\|g(\omega)\|_{X} \lambda$-a.e. and for all $n \in \mathbb{N}$ (see [7, Theorem 6, p. 4]). Hence for every $\alpha>0$, we have that $\varphi^{*}\left(\alpha \| f_{n}(\omega)-\right.$ $\left.g(\omega) \|_{X}\right) \rightarrow 0 \lambda$-a.e. and $\varphi^{*}\left(\alpha\left\|f_{n}(\omega)-g(\omega)\right\|_{X}\right) \leq \varphi^{*}\left(2 \alpha\|g(\omega)\|_{X}\right) \lambda$-a.e. and for all 
$n \in \mathbb{N}$, where $\int_{\Omega} \varphi^{*}\left(2 \alpha\|g(\omega)\|_{X}\right) d \lambda<\infty$. By the Lebesgue dominated convergence theorem, $\int_{\Omega} \varphi^{*}\left(\alpha\left\|f_{n}(\omega)-g(\omega)\right\|_{X}\right) d \lambda \rightarrow 0$ and this means that $\left\|f_{n}-g\right\|_{\varphi^{*}}^{0} \rightarrow 0$.

For each $n \in \mathbb{N}$, let $S_{n}: L^{\varphi} \rightarrow X$ be a linear operator defined by

$$
S_{n}(u):=\int_{\Omega} u(\omega) f_{n}(\omega) d \lambda \text { for all } u \in L^{\varphi}
$$

Note that the range of each $S_{n}$ is contained in the span of finite set of values of $f_{n}$. Therefore $S_{n}$ is compact and by the Hölder's inequality for each $u \in L^{\varphi}$, we have

$$
\begin{gathered}
\left\|\left(S_{n}-S_{g}\right)(u)\right\|_{X}=\left\|\int_{\Omega} u(\omega)\left(f_{n}(\omega)-g(\omega)\right) d \lambda\right\|_{X} \\
\leq \int_{\Omega}|u(\omega)|\left\|f_{n}(\omega)-g(\omega)\right\|_{X} d \lambda \leq\|u\|_{\varphi}\left\|f_{n}-g\right\|_{\varphi^{*}}^{0} .
\end{gathered}
$$

It follows that $\left\|S_{n}-S_{g}\right\| \rightarrow 0$, so $S_{g}$ is a compact operator.

(ii) In view of (i) and Proposition $3.2 S_{g}$ is $\gamma_{\varphi}$-compact. Using Theorem 3.3 we obtain that (ii) holds.

(iii) Let $s=\sum_{i=1}^{k} c_{i} \mathbb{1}_{A_{i}} \in \mathcal{S}(\Sigma)$ and $\|s\|_{\varphi} \leq 1$. Then

$$
\sum_{i=1}^{k}\left|c_{i}\right|\left\|m\left(A_{i}\right)\right\|_{X}=\sum_{i=1}^{k}\left|c_{i}\right| \int_{A_{i}}\|g(\omega)\|_{X} d \lambda=\int_{\Omega}|s(\omega)|\|g(\omega)\|_{X} d \lambda \leq\|g\|_{\varphi^{*}}^{0}
$$

and hence $|m|_{\varphi^{*}}(\Omega) \leq\|g\|_{\varphi^{*}}^{0}$. Note that $m=m_{S_{g}}$. Hence using Lemma 2.1 we have that $\left|m_{\varphi^{*}}\right|(A)=\left\|\mathbb{1}_{A} g\right\|_{\varphi^{*}}^{0}$ for $A \in \Sigma$.

(iv) This follows from (iii) because $g \in E^{\varphi^{*}}(X)$.

Let $i_{\infty}: L^{\infty} \rightarrow L^{\varphi}$ denotes the inclusion map. Note that $i_{\infty}$ is $\left(\sigma\left(L^{\infty}, L^{1}\right)\right.$, $\left.\sigma\left(L^{\varphi}, L^{\varphi^{*}}\right)\right)$-continuous, and it follows that $i_{\infty}$ is $\left(\tau\left(L^{\infty}, L^{1}\right), \tau\left(L^{\varphi}, L^{\varphi^{*}}\right)\right)$-continuous (see [8, Theorem 8.6.1]). Since $\gamma_{\varphi} \subset \tau\left(L^{\varphi}, L^{\varphi^{*}}\right)$, we obtain that $i_{\infty}$ is $\left(\tau\left(L^{\infty}, L^{1}\right), \gamma_{\varphi}\right)$ continuous.

As a consequence of Theorem 3.4 and Theorem 2.5, we can show that every $\tau\left(L^{\infty}, L^{1}\right)$-nuclear operator $T: L^{\infty} \rightarrow X$ admits a factorization through some Orlicz space $L^{\varphi}$.

Corollary 3.5 Assume that $T: L^{\infty} \rightarrow X$ is a $\tau\left(L^{\infty}, L^{1}\right)$-nuclear operator. Then there exists a Young function $\varphi$ such that $T=S \circ i_{\infty}$, where

(i) $S: L^{\varphi} \rightarrow X$ is a Bochner representable and $\gamma_{\varphi}$-compact linear operator.

(ii) $\left|m_{S}\right|_{\varphi^{*}}\left(A_{n}\right) \rightarrow 0$ if $\lambda\left(A_{n}\right) \rightarrow 0$. 
Proof (i) In view of Theorem 2.5 the representing measure $m_{T}: \Sigma \rightarrow X$ has the Radon-Nikodym property with respect to $\lambda$, that is, $m_{T}=g \lambda$, where $g \in L^{1}(X)$. Hence according to Theorem 3.4 there exists a Young function $\varphi$ such that $g \in$ $E^{\varphi^{*}}(X)$ and an operator $S: L^{\varphi} \rightarrow X$ defined by

$$
S(u):=\int_{\Omega} u(\omega) g(\omega) d \lambda \text { for all } u \in L^{\varphi},
$$

is $\gamma_{\varphi}$-compact. Note that for $u \in L^{\infty}, T(u)=\int_{\Omega} u d m_{T}=\int_{\Omega} u(\omega) g(\omega) d \lambda=$ $S(u)$.

(ii) Since $m_{S}(A)=\int_{A} g(\omega) d \lambda$ for all $A \in \Sigma$, using Theorem 3.4 we have that $\left|m_{S}\right|_{\varphi^{*}}\left(A_{n}\right) \rightarrow 0$ if $\lambda\left(A_{n}\right) \rightarrow 0$.

Acknowledgements The author wishes to express his thanks to the referee for remarks and suggestions leading to an improvement of the paper.

Open Access This article is licensed under a Creative Commons Attribution 4.0 International License, which permits use, sharing, adaptation, distribution and reproduction in any medium or format, as long as you give appropriate credit to the original author(s) and the source, provide a link to the Creative Commons licence, and indicate if changes were made. The images or other third party material in this article are included in the article's Creative Commons licence, unless indicated otherwise in a credit line to the material. If material is not included in the article's Creative Commons licence and your intended use is not permitted by statutory regulation or exceeds the permitted use, you will need to obtain permission directly from the copyright holder. To view a copy of this licence, visit http://creativecommons.org/licenses/by/4.0/.

\section{References}

1. Cooper, J.B.: The strict topology and spaces with mixed topologies. Proc. Amer. Math. Soc. 30(3), 583-592 (1971)

2. Cristescu, R.: Topological Vector Spaces. Noordhoff Int. Publ, Leyden (1977)

3. Diestel, J.: Sequences and Series in Banach Spaces. Graduate Texts in Math, vol. 92. Springer-Verlag, New York (1984)

4. Diestel, J., Uhl, J.J.: Vector Measures, Math. Surveys, vol. 15. American Mathematical Society, Providence, RI (1977)

5. Diestel, J., Fourie, J., Swart, J.: The Metric Theory of Tensor Products. Grothendieck's resume revisited. American Mathematical Society, Providence (2008)

6. Dinculeanu, N.: Vector Measures. Pergamon Press, New York (1967)

7. Dinculeanu, N.: Vector Integration and Stochastic Integration in Banach Spaces. A Wiley-Interscience Publication, New York (2000)

8. Edwards, R.E.: Functional Analysis. Theory and Applications. Holt, Rinehart and Winston, New York (1965)

9. Fremlin, D.H.: Topological Riesz Spaces and Measure Theory. Cambridge University Press, LondonNew York (1974)

10. Grothendieck, A.: Sur les espaces (F) et (DF). Summa Brasil. Math. 3, 57-123 (1954)

11. Grothendieck, A.: Produits tensoriels topologiques et espaces nucléaires. Mem, vol. 16. American Mathematical Society, Providence (1955)

12. Jarchow, H.: Locally Convex Spaces. Teubner, Stuttgart (1981)

13. Kantorovitch, L.V., Akilov, G.P.: Functional Analysis. Pergamon Press, Oxford-Elmsford, New York (1982)

14. Lin, P.-K.: Köthe-Bochner Function Spaces. Birkhaüser, Boston (2003) 
15. Nowak, M.: Some equalities among Orlicz spaces II. Bull. Pol. Acad. Sci. Math. 34(11-12), 675-687 (1986)

16. Nowak, M.: Compactness of Bochner representable operators on Orlicz spaces. Positivity 13, 193-199 (2009)

17. Nowak, M.: Absolutely continuous operators on function spaces and vector measures. Positivity 17, 525-533 (2013)

18. Pietsch, A.: Nuclear Locally Convex Spaces, Ergebnisse der Mathematik und ihrer Grenzebiete, vol. 66. Springer, Berlin (1972)

19. Rao, M.M., Ren, Z.D.: Theory of Orlicz Spaces. Marcel Dekker, New York (1991)

20. Ruess, W.: [Weakly] compact operators and DF-spaces. Pacific J. Math. 98, 419-441 (1982)

21. Ruston, A.F.: On the Fredholm theory of integral equations for operators belonging to the trace class for a general Banach space. Proc. London Math. Soc. 55(2), 109-124 (1951)

22. Ryan, R.: Introduction to the Tensor Products of Banach Spaces. Springer Monographs in Math. Springer-Verlag, London (2002)

23. Schaefer, H.H.: Topological Vector Spaces. Springer, New York, Heidelberg, Berlin (1971)

24. Swartz, C.: An operator characterization of vector measures which have Radon-Nikodym derivatives. Math. Ann. 202, 77-84 (1973)

25. Wiweger, A.: Linear spaces with mixed topology. Studia Math. 20, 47-68 (1961)

26. Yosida, K.: Functional Analysis, 4th edn. Springer-Verlag, Berlin, Heidelberg, New York (1974)

27. Zaanen, A.C.: Riesz Spaces II. North-Holland Publ. Comp, Amsterdam (1983)

Publisher's Note Springer Nature remains neutral with regard to jurisdictional claims in published maps and institutional affiliations. 